\title{
Socicty Migroxts.
}

NEW YORK NEUROLOGICAL SOCIETY.

Stated Mceting, held at the New York Acadiny of Medicine, Tuesday caining, Warch $7,1893$.

Dr. M. Allex Stakr, President, in the chair.

\section{CLONIC SPASM OF THE MUSCLES OF MASTI- CATION.}

Dr. Frederick Peterson presented this case. The patient was a woman, aged 57 . Six years ago she had all her upper teeth removed and artificial ones put in. The first set did not fit well, and a new one was substituted. The work about the mouth, and the necessity for keeping her mouth open for long periods of time while in the dentist's chair resulted in the development of this spasm. When she is sitting quietly, not using the jaw muscles, there is a continuous clonic spasm of the masseters, temporals, and pterygoids. The jaw opens and shuts slightly, and moves from side to side. She is tired and worn out trying to keep her teeth together. The chief difficulty, however, is when she attempts to speak; then the mouth opens widely and there is a sub-luxation of the jaw downwards and forwards from the glenoid cavity. During the first six months the mouth would not close at all, excepting at night, when the spasm relaxed.

Dr. Peterson said that while tonic spasm of the masticatory muscles, or trismus, is quite a common symptom, the condition presented in this case is very rare. As regards treatment, atropia, hyoscine, conium and electricity were used perseveringly without any special effect. Latterly, the sulphate of duboisine, in doses of $\frac{1}{20}$ of a grain, three times daily, has afforded much relief by quieting the spasmodic movements almost wholly at times. In addition, she wears an apparatus made especially for her which keeps the jaw closed, and allows her to talk between her teeth without the uncomfortable 
tonic spasm of the depressors of the jaw, although the clonic movements of the masseters and pterygoids may keep on as before. The movements cease at night. The affection has lasted nearly seven years.

The PRESIIENT stated that he saw the patient four years agro. He confirmed Dr. Peterson's history of the case.

Dr. Wilitialr M. LESZYNSKY said he has seen a case of clonic spasm of both platysma, following prolonged work upon the lower jaw of the patient by a dentist. The spasm in that case only lasted three or four days. Slight spasm of the jaw muscles after dental operations is not uncommon.

\section{A CASE OF RYTHMICAL SPASM OF THE EX- TREMITIES.}

Dr. EIIWAR], D. FISHER presented this case. The patient was a woman, aged 43 years. Previous history negative. The patient has always enjoyed good health. About one year ago she began to have these attacks of rythmical spasm of the extremities. The spasm is sometimes confined to one or both arms, or to the lower extremities, or the motion may involve all the limbs at the same time. The attacks come on by themselves and last about five minutes, A number of such attacks may occur during the day, or she may remain free from them for a day or two. 'The muscles are becoming more rigid and it is very difficult to bend the elbow or the arm. The woman's general symptoms otherwise are negative. She complains of fatigue. The muscles of the arm are becoming markeāly developed. Various methods of treatment, hypnotism included, have been employed without apparent effect. Under hypnotism she had all four cxtremities moving violently. Her eye-sight is apparently normal. It seems to be impossible for her to control the spasm. There is no analgæesia. Dr. Fisher said he considered hysteria to be the basis of the attacks.

Dr. PETERSON, in reply to a question, said he did not think that duboisine would prove of much value in this case, which was probably hysterical.

Dr. Mari Putram Jacobi referred to the cases of head jerking in children; the rythmical movements sometimes seen in such cases she considered rather analogous to those in the case presented. 